Research Article

\title{
Impact of UAV Delivery on Sustainability and Costs under Traffic Restrictions
}

\author{
Yuyu Li $\mathbb{D}^{1},{ }^{1}$ Wei Yang, ${ }^{2}$ and Bo Huang $\mathbb{D}^{3}$ \\ ${ }^{1}$ School of Economics and Management, Chongqing Normal University, Chongqing 400047, China \\ ${ }^{2}$ College of Computer and Information Science, Chongqing Normal University, Chongqing 400047, China \\ ${ }^{3}$ School of Economics and Business Administration, Chongqing University, Chongqing 400044, China
}

Correspondence should be addressed to Bo Huang; huangbo@cqu.edu.cn

Received 5 January 2020; Revised 9 June 2020; Accepted 7 July 2020; Published 8 August 2020

Academic Editor: Mosè Gallo

Copyright (c) 2020 Yuyu Li et al. This is an open access article distributed under the Creative Commons Attribution License, which permits unrestricted use, distribution, and reproduction in any medium, provided the original work is properly cited.

\begin{abstract}
Compared with traditional vehicles delivery, unmanned aerial vehicle (UAV) delivery can reduce energy consumption and greenhouse gas emissions, which benefits environmental sustainability. Besides, UAVs can overcome traffic restrictions, which are the big obstacle in parcel delivery. In reality, there are two kinds of most popular traffic restrictions, vehicle-type restriction, and half-side traffic. We propose a mixed-integer (0-1 linear) green routing model with these two kinds of traffic restrictions for UAVs to exploit the environmental aspects of the use of UAVs in logistics. A genetic algorithm is proposed to efficiently solve the complex routing problem, and an experimental analysis is made to illustrate and validate our model and the algorithm. We found that, under both these two traffic restrictions, UAV delivery can accomplish deliveries that cannot be carried out or are carried out at much higher costs by vehicles only and can always effectively save costs and cut $\mathrm{CO}_{2}$ emissions, which is environmentally friendly. Furthermore, UAV delivery saves more cost and cuts more $\mathrm{CO}_{2}$ emission under the first kind of traffic restriction than that under the second.
\end{abstract}

\section{Introduction}

Brick-and-mortar retail stores being gradually replaced by ecommerce saves energy consumption for heating and lighting in stores and warehouses. However, the rapid development of e-commerce dramatically increases the demands on logistics or parcel delivery, which is mainly carried out by traditional vehicles. As a result, energy consumption and $\mathrm{CO}_{2}$ emission are increased [1].

With the development of unmanned aerial vehicles (UAVs), they become one of the best choices improving the "last-mile" delivery of products to consumers, both economically and environmentally, due to their significant performances in saving costs and energy. Unfortunately, current UAVs have two big shortcomings: both delivery range (distance and flight time) and capacity (weight and size) are limited. Therefore, they often cannot deliver all packages themselves in a single trip, and the most reasonable way to use a UAV is to pair it with a traditional vehicle. In this "UVA-vehicle mode," a UAV is loaded on the roof of the vehicle, and the driver guides the UAV to carry the package to the destination. At the same time of UAV delivery, the vehicle serves other customers. Although the UAV only serves part of customers, this mode of package delivery makes a best use of the advantages of UAVs and avoids their disadvantages. In other words, this mode normally delivers all packages in one signal trip with much lower time, cost, and energy consumption.

Besides, in many big cities, because of heavy traffic jam or severe air pollution from vehicle exhaust, vehicle-type restriction is implemented on vehicles. Delivery vehicles are forbidden to run on some roads during some periods. Besides, during the maintenance of some roads, only one lane is available, and vehicles traveling in opposite directions have to take turns on the road with a much lower speed. This is half-side traffic, which is other most popular traffic restriction. Traffic restrictions bring big troubles to package delivery service and even some "impossible missions" to 
delivery by vehicles only, which can be normally overcome through UAV delivery.

In order to make the most use of the advantages of this "UAV-vehicle mode" and better understand the economic and environmental impact of using UAVs on packages delivery, we should optimize the routing plan of "UVAvehicle mode." To this end, we propose a mixed-integer (0-1 linear) green routing model with traffic restrictions for UAVs and design a genetic algorithm (GA) to effectively solve the routing plan model. Finally, we make an experimental analysis to illustrate and validate our model and algorithm, as well as better understand the environmental benefits of delivering with UAVs.

The rest of this paper is organized as follows: we review the literature on "green routing" and routing problem with UAVs in Section 2; we propose a UAV-vehicle routing model with traffic restrictions and design a genetic algorithm to effectively solve the model in Sections 3 and 4, respectively; an experimental analysis is made in Section 5; and conclusions are drawn in Section 6.

\section{Literature Review}

Since the emergence of "truck dispatching problem," vehicle routing problem (VRP) has been a hot research field [2]. And many heuristic algorithms were developed to solve VRP [3]. Our paper is mainly relevant to two topics: green vehicle routing problem and routing with UAVs, which are reviewed below.

2.1. Green Vehicle Routing Problem. A series of studies show that, for the logistics, transportation accounts for about $90 \%$ of the energy consumption of the whole logistics. Therefore, reducing $\mathrm{CO}_{2}$ emissions is the core of the green vehicle routing problem (GVRP). As early as 1985, Cermak and Takeda [4] outlined criteria for simulating atmospheric boundary layer and physical simulation of source characteristics in boundary layer wind tunnels and studied the air problem in urban environment. Their work led subsequent scholars to study logistics and carbon emissions.

Pradenas et al. [5] studied the vehicle path problem for the energy required for each path from the perspective of vehicle distance and estimated the load and distance between customers to achieve the goal of reducing fuel consumption and carbon emission. Qian [6] developed routing and scheduling model for fleets of transport vehicles to minimize fuel emissions in the speed-dependent road network from the perspective of vehicle speed. In this paper, the route of each vehicle needs to be clarified, the speed of vehicles on different roads on their respective paths is regarded as a decision variable, and the time insertion algorithm is given for a single path. Finally, a tabu search algorithm based on column generation is proposed to solve this problem. Kwon et al. [7] built a vehicle path model that minimizes energy consumption and pollution emissions with time windows from the perspective of vehicle load. Their simulation process is based on the actual route of motor vehicles, and their method can save more than $6.9 \%$ of fuel compared with the existing method. Although these papers only studied GVRP without UAVs, we can refer to their methods of calculating delivery cost and fuel consumption in establishing our model.

2.2. Routing with UAVs. The "last kilometer problem" is always the bottleneck of logistics distribution due to traffic conditions, distribution personnel, customer acceptance location, and other factors. In recent years, UAVs were introduced into logistics to reduce costs and solve the "last kilometer problem."

In 2013, Jeff Bezos, CEO and founder of Amazon.com, announced on "60 minutes" that UAVs could be used to speed up the delivery of packages to consumers. Subsequently, routing with UAVs became a hot topic in VRP field [8]. D'Andrea [9] calculated the energy consumption and the cost of high-end lithium ion batteries in the highperformance mobile delivery of UAVs based on the principle of first computability and systematically analyzed the economy of UAV technology and future applications. Zhi [10] designed a two-stage hitch-matching algorithm to serve the UAV driving equipment, which greatly reduced the logistics cost and improved the efficiency. Weng [11] made a systematic evaluation of UAV logistics from the aspects of laws and regulations, technical safety, audience preference, operation safety, and use cost. Ma et al. [12] established a model of UAV flight stability index and various factors and analyzed and studied the main factors influencing the flight stability of UAVs through Matlab.

The feature of our "UVA-vehicle mode" is that UAV departures from the roof of the vehicle to deliver package; meanwhile, the vehicle serves other customers, which improves logistics efficiency and reduces energy consumption. Chiang et al. [2] proposed a UAV-vehicle model and a GA to study the economic and environmental impact of using UAVs on package delivery. Murray and Chu [13] conducted relevant research, proposing two mixed-integer linear programming formulas for unmanned delivery problems, as well as two simple but effective heuristic solutions to satisfy the scheduling of UAVs and delivery trucks. Ha et al. [14] considered a new variant of TSP-D in which the objective is to minimize operational costs including total transportation cost and one created by waste time a vehicle has to wait for the other. They formulated problem and proposed two algorithms, TSP-LS and GRASP, to solve the problem. Wang et al. [15] pose a number of questions in order to study the maximum savings that can be obtained from using drones and derived a number of worst-case results. Poikonen et al. [16] studied the UAV-vehicle delivery routing with the aim of minimizing the total operation time. Pugliese and Guerriero [17] analyzed the delivery process with drones, by taking into account the total transportation cost, under the assumption that all customers should be served within their time window.

Although the above papers studied UAV-vehicle routing problems under different circumstances, they failed to 
research the use of UAVs and its effect on relieving one of the greatest constraints in parcel delivery and traffic restrictions [18]. In fact, there are two kinds of traffic restrictions, which allow the implementation of our UAVvehicle mode but greatly affect the cost, energy consumption, and efficiency of parcel delivery. The first is vehicle-type restriction, mainly on delivery vehicles. For example, during the maintenance, all vehicles except public transportation were banned to run across Shimen Bridge, located in Chongqing, China. Under this circumstance, the delivery vehicle has to choose other way or use a UAV. The second is half-side traffic, under which the delivery vehicle can choose this road but may have to wait to pass through it. During its waiting, the engine is still operating and consuming gasoline, causing a higher cost and $\mathrm{CO}_{2}$ emission [19].

2.3. Algorithms for VRP. To solve VRP, we should design an algorithm. Generally, there are two categories of algorithms: exact algorithm and approximation algorithm [20]. The advantage of exact algorithm is that it can find the accurately optimal solution of VRP, but it is only suitable for VRP of small scale [21]. When the scale is large, it is mainly applied to obtain the initial solution for the approximation algorithm [22].

Approximation algorithm can be roughly divided into two categories: heuristic algorithm, which consists of constructive heuristic algorithm and improved heuristic algorithm, as well as meta-heuristic algorithm [23]. The advantage of constructive heuristic algorithm is that it is simple and easy to understand, but the solutions found may be far from the optimal solutions. Therefore, it is no longer used for solving VRP alone but combined with the improved heuristic algorithm, being used to generate initial solutions [24]. Improved heuristic algorithm can obtain better solutions from initial solutions generated by constructive heuristic algorithm through neighborhood search. Its advantage is that the probability of obtaining the optimal solution is high, but the operation time may be very long [25].

The biggest disadvantage of heuristic algorithm is that it is easy to fall into local optimal. In order to overcome it, there appeared a variety of meta-heuristic algorithms with the advantage of jumping out of the local optimal and seek the global optimal, such as genetic algorithm, simulated annealing algorithm, and tabu search algorithm [26, 27]. Among meta-heuristic algorithms, the genetic algorithm is popularly applied for solving VRP, including VRP with UAVs $[28,29]$. However, the mode of UAV delivery in these studies is that the truck is just used as a mobile base, which is very different with ours. Therefore, these genetic algorithms cannot solve the key issue in our mode, that is, which customer is served by the UAV and how many and which customers should be served by the vehicle.

The main contributions of this paper are as follows. Firstly, taking into account two kinds of traffic restrictions which affect parcel delivery, we propose a mixed-integer (0-1 linear) UAV-vehicle routing model with traffic restrictions to incorporate environment aspects to study the impacts of using UAVs for package delivery under these two circumstances and analyze the efficiency of UAV delivery to reduce cost and energy consumption by comparing the results under these two circumstances and that without UAVs. Secondly, we develop a genetic algorithm to effectively solve the model and analyze the impact of UAV delivery. In our GA, we make improvement and contribution in the generation of initial solution and grouping customers to determine which customer is served by the UVA and which customer(s) should be served by the vehicle during UAV delivery.

\section{Problem Description and the Model}

3.1. Problem Description. In an urban area, a delivery company uses vehicles equipped with UAVs to deliver parcels from a warehouse to customers in its delivery area. Each customer has only one parcel waiting to be delivered. This UVA-vehicle mode is as follows. Every vehicle is equipped with a UAV. As both delivery range (distance and flight time) and capacity (weight and size) of the UAV are limited, it only delivers parts of the parcels, but the vehicle can deliver all the parcels. Each UAV may depart from its vehicle at a location of a customer or the warehouse and carry a parcel to one and only one customer. Then, the UAV returns to its vehicle to reload a parcel and recharge or swap batteries, which is instantaneous. While the UAV delivers the parcel, the vehicle carries out its delivery to one or several customers. Therefore, the UAV returns to its vehicle at a different customer location. As a matter of course, if the vehicle arrives at the customer location, where the UAV is retrieved, it waits for the UAV, and vice versa. For the sake of the safety of the UAV, if the UAV arrives before the vehicle, it hovers in the air waiting for the vehicle.

In the delivery area of the warehouse, there may be traffic restrictions on the vehicles. Generally, there are two kinds of traffic restrictions, under which this UAV-vehicle mode is practicable but greatly affected. The first is vehicle-type restriction, under which delivery vehicles are forbidden to run through some paths and have to choose other way or use a UAV. The second is half-side traffic, under which only one lane of a road is available, and vehicles traveling in opposite directions can only take turns on this road. Under this circumstance, the delivery vehicle can run on this road but may have to wait for its turn with the operating engine and raise the variable cost and $\mathrm{CO}_{2}$ emission.

According to the regulation on working hours, the service time of every pair of UAV and vehicle is 8 hours. Therefore, it is probable that more than one pair of UAV and vehicle are needed.

There are two goals of routing the UAV-vehicle. The first is minimizing the total cost, and the alternative is minimizing the energy consumption (or $\mathrm{CO}_{2}$ emission). The total cost consists of fixed cost whenever a pair of UAV-vehicle is used, as well as variable cost, which is the function of unit route cost, travel distance, and gross weight (empty weight of the vehicle or UAV plus the payload). The total $\mathrm{CO}_{2}$ emission of a vehicle is the function of weighted average emission rate of vehicles, travel distance, and gross weight, and that of a UAV is the function of $\mathrm{CO}_{2}$ emission rate of 
generating per watt-hour, average energy requirement of UAV, travel distance, and gross weight.

It should be noted that the two kinds of traffic restrictions may simultaneously exist, but as we analyze the impact of UAV delivery under every kind of traffic restriction, they are in our models separately.

3.2. The Model. According to Laporte [30], there are three ways to formulate the vehicle routing problem (VRP), including the simple set division formula of VRP first proposed by Balinski and Quandt in 1964, the commodity flow formula of Shlifer and Graves in 1979, and the two-index vehicle flow formula of Laporte and Norbert in 1983. In our study, the GVRP problem is mainly involved, and the weight of vehicles, commodities, and drones in the whole route needs to be considered. Therefore, we choose to continue to expand the commodity flow formula, which is clearly expressed and promotes the development of heuristic algorithm.

The basic GVRP problem is as follows: both the UAV and the vehicle can travel back and forth on any available path. Therefore, an undirected graph $R=(M, N)$ is given, where $N$ is the set of edges with nonnegative routing costs, $\{i, j\} \in N$ represents the edges from node $i$ to node $j . M=\{0$, $1, \ldots, n\}$ is the set of all nodes with a total of $n$ customers, and the warehouse is labeled as 0 . Therefore, $U=M \backslash\{0\}$ is the set of customer nodes. The distance between each node is $L_{i j}$. Every customer has a demand for $P_{i}$ units; for calculating energy consumption, we measure demand in units of weight.

In our UAV-vehicle mode, each vehicle with a payload (weight) capacity of $Q^{V}$ is equipped with a UAV. Each pair of a vehicle and a UAV has a fixed cost $C^{F}$, as well as a variable route cost, which is a function of range and gross weight (see next paragraph). The unit route cost $C^{U}$ of the UAV is expected to be much lower than that of the vehicle $C^{V}$. However, the payload (capacity) $Q^{U}$ of the UAV and the distance $L^{U}$ and the time $T^{U}$ that the UAV can fly in the air are limited. Note that the time limit may include the time the UAV has to wait for the vehicle before landing. The distance between the vehicle and the UAV may be different; in fact, another potential advantage of UAVs is that they may choose more efficient routes than vehicles. For example, vehicles need to follow the Manhattan metric, while UAVs can use Euclidean distances. Therefore, we distinguish between the distance from $i$ to $j$ by the vehicle, $L_{i j}^{V}$, and that by the UAV, $L_{i j}^{U}$. It is assumed that the UAV will serve only one customer before returning to the vehicle but can serve other customers after returning the vehicle to reload and replace batteries.

If the vehicle starts from the node $i$ to the node $j,\{i, j\} \in$ $N$, let $N_{i j}$ be 1 ; otherwise, let it be 0 . Let $M_{i j}$ be the vehicle payload weight for edge $\{i, j\} \in N$. Obviously, $M_{i j}=0$, if the vehicle does not run from the node $i$ to the node $j$. Finally, if the UAV leaves the vehicle at node $i$, serves the $q_{j}$ unit required by customer $j$, and returns to node $k$ and lands on the roof of the vehicle, then $Z_{i j k}=1$ and the flight time is $T_{i j k}$; otherwise, $Z_{i j k}=0$.
In the delivery area of the warehouse, there separately exist two kinds of traffic restrictions on the vehicles. Under the vehicle-type restriction (labeled as "circumstance 1"), there are some edges where vehicles are forbidden to drive. Let $\bar{N}_{1}$ be the set of these edges, then, for any $\{i, j\} \in \bar{N}_{1}$, $N_{i j}=0$. Under half-side traffic (labeled as "circumstance 2"), the average speed, variable cost, and $\mathrm{CO}_{2}$ emission of the vehicle on these edges are raised by $R^{S}, R^{C}$, and $R^{E}$ times, respectively. Let $\bar{N}_{2}$ be the set of these edges, then $R_{i j}^{S}=$ $R_{i j}^{C}=R_{i j}^{E}=1$ for any $\{i, j\} \notin \bar{N}_{2}$ and $R_{i j}^{S}<1, R_{i j}^{C}>1$, and $R_{i j}^{E}>1$ for any $\{i, j\} \in \bar{N}_{2}$.

Franzese and Davidson [31] pointed out that the increase in total vehicle weight has a certain impact on its fuel efficiency, and fuel is an important component of variable transportation costs, so they added relevant factor of fuel to GVRP. Bateman et al. [32] pointed out that the carbon footprint of transportation emissions can be estimated by transportation weight and distance traveled.

On the route from node $\mathrm{i}$ to $\mathrm{j}$, the total vehicle weight is $W_{i j}$, including the empty weight of the vehicle $\mathrm{W}^{\mathrm{V}}$, plus the payload weigh $M_{i j}$, and the UAV weight $W^{U}$ if the UAV is on the vehicle. The weight can be expressed as a nonlinear function:

$$
W_{i j}=W^{V} N_{i j}+M_{i j}+W^{U} N_{i j}\left(1-\sum_{h \in U} Z_{i h j}\right) .
$$

The first term on the right hand of (1) is the vehicle empty weight when running from $i$ to $j$, the second is the weight of packages on the vehicle from $i$ to $j$, and the third is the weight of the UAV if it is on the vehicle from $i$ to $j$. Another linear formula is

$$
W_{i j} \geq W^{V} N_{i j}+M_{i j}+W^{U}\left(N_{i j}-\sum_{h \in U} Z_{i h j}\right) .
$$

Although (1b) may generate negative values for edges that do not exist in the solution, these values will be 0 because of (21). To calculate the vehicle route cost, we use the unit cost, $C^{V}$, time distance, $L_{i j}^{V}$, and time total vehicle weight, $W_{i j}$ (note that $C^{V}$ is calculated in dollars per poundmile and can determined by regression analysis).

On the route of the UAV flying from $i$ to $j$ to $k$, the total weights of the UAV are different. On the route of the UAV flying from $i$ to $j$, the total weight of the UAV is

$$
W_{i j}^{U}=\left(W^{U}+q_{j}\right) Z_{i j k} \text {. }
$$

On the route of the UAV flying from $j$ to $k$, the total weight of the UAV is

$$
W_{j k}^{U}=W^{U} Z_{i j k}
$$

To calculate the vehicle route cost, we use the unit cost, $C^{U}$, time distance, $L_{i j}{ }^{U}$ and $L_{j k}{ }^{U}$, and total UAV weight, $W_{i j}^{U}$ and $W_{j k}^{U}$.

There are two ways to analyze the impact of UAV delivery. The first is the benefit on total $\mathrm{CO}_{2}$ emission. The total $\mathrm{CO}_{2}$ emission of a vehicle can be calculated by the ways of Goodchild and Toy [33] as follows: 


$$
\begin{aligned}
& E_{1}^{V}=\operatorname{WAER} \sum_{i \in M} \sum_{j \in M} L_{i j}^{V} \times W_{i j}, \\
& E_{2}^{V}=\operatorname{WAER} \sum_{i \in M} \sum_{j \in M} R_{i j}^{E} \times L_{i j}^{V} \times W_{i j},
\end{aligned}
$$

where WAER is the weighted average emission rate of vehicles. (3a) is the formulation of a vehicle's total $\mathrm{CO}_{2}$ emission under circumstance 1 , and $(3 \mathrm{~b})$ is that under circumstance 2. Obviously, in (3a), $W_{i j}=0$ for any $\{i$, j\} $\in \bar{N}_{1}$.

The total $\mathrm{CO}_{2}$ emission of UAV is calculated as

$$
E^{U}=\operatorname{PGFER} \times \operatorname{AER} \sum_{i \in M} \sum_{j \in M} \sum_{k \in M}\left(L_{i j}^{U} W_{i j}^{U}+L_{j k}^{U} W_{j k}^{U}\right) \text {, }
$$

where PGFER is the $\mathrm{CO}_{2}$ emission rate per watt-hour (Wh) of the power generation facilities in the generation of electricity for using the UAV and AER is the average energy requirement of UAV in Wh per pound-mile.

Therefore, the environmental goals of minimizing total $\mathrm{CO}_{2}$ emission under circumstances 1 and 2 are, respectively,

$$
\begin{aligned}
& \operatorname{minimize}\left(E_{1}^{V}+E^{U}\right), \\
& \operatorname{minimize}\left(E_{2}^{V}+E^{U}\right) .
\end{aligned}
$$

The alternative way is examining its impact on the traditional objective of minimizing total cost, TC, under circumstances 1 and 2, including the fixed cost of the pair of a UAV and a vehicle and the variable route cost of the vehicle and the UAV:

$$
\begin{aligned}
& \mathrm{TC}_{1}=C^{F} \sum_{j \in U} N_{0, j}+C^{V} \sum_{i \in M} \sum_{j \in M} L_{i j}^{V} \times W_{i j}+C^{U} \sum_{i \in M} \sum_{j \in U} \sum_{k \in M}\left(L_{i j}^{U} W_{i j}^{U}+L_{j k}^{U} W_{j k}^{U}\right), \\
& \mathrm{TC}_{2}=C^{F} \sum_{j \in U} N_{0, j}+C^{V} \sum_{i \in M} \sum_{j \in M} R_{i j}^{C} \times L_{i j}^{V} \times W_{i j}+C^{U} \sum_{i \in M} \sum_{j \in U} \sum_{k \in M}\left(L_{i j}^{U} W_{i j}^{U}+L_{j k}^{U} W_{j k}^{U}\right) .
\end{aligned}
$$

Therefore, the traditional goals of minimizing total cost under circumstances 1 and 2 are, respectively, as follows:

$$
\text { minimize } \mathrm{TC}_{1} \text {, }
$$

minimize $\mathrm{TC}_{2}$
Let $V_{i j}^{V}$ and $V_{i j}^{U}$, respectively, be the average speed of the vehicle and the UAV from node $i$ to node $j$ (mile/hour). The constraint set of the two goals can be expressed as follows. It should be noted that the same set of constraints are applied to the above two objectives and is solved twice:

$$
\begin{gathered}
\sum_{j \in M} N_{i j}+\sum_{h \in M} \sum_{k \in M} Z_{h i k}=1, \quad \forall j \in U, \\
\sum_{i \in M} N_{i j}+\sum_{h \in M} \sum_{k \in M} Z_{h j k}=1, \quad \forall j \in U, \\
\sum_{j \in U} N_{0 j}=\sum_{i \in U} N_{i 0}, \\
\sum_{j \in M} M_{j i}-\sum_{j \in M} M_{i j}+\sum_{h \in M} \sum_{k \in M} q_{i} Z_{h i j}-\sum_{j \in M} \sum_{k \in M} q_{j} Z_{i j k}=q_{i}, \quad \forall i \in U, \\
N_{i k} \geq \sum_{j \in M} Z_{i j k}, \quad \forall i, k \in M, \\
\sum_{j \in M} q_{j}+W^{U} \leq Q^{V}, \\
q_{j} \sum_{i \in M} \sum_{k \in M} Z_{i j k} \leq Q^{U}, \quad \forall j \in U, \\
\left(L_{i j}^{U}+L_{j k}^{U}\right) Z_{h i k} \leq L^{U}, \quad \forall i, j, k \in M,
\end{gathered}
$$




$$
\begin{aligned}
& T_{i j k} \geq\left(\frac{L_{i j}^{U}}{V_{i j}^{U}}+\frac{L_{j k}^{U}}{V_{j k}^{U}}\right) Z_{h j k}, \quad \forall i, k \in M, \forall j \in U, \\
& T_{i j k} \geq \frac{L_{i k}^{V}}{V_{i k}^{V}} N_{i k}, \quad \forall i, k \in M, \forall j \in U, \\
& T_{i j k} \geq \frac{L_{i k}^{V}}{R_{i k}^{S} V_{i k}^{V}} N_{i k}, \quad \forall i, k \in M, \forall j \in U, \\
& T_{i j k} \leq T^{U}+G\left(1-Z_{h j k}\right), \quad \forall i, k \in M, \forall j \in U, \\
& W_{i j} \geq W^{V} N_{i j}+M_{i j}+W^{U}\left(N_{i j}-\sum_{h \in U} Z_{i h j}\right), \quad \forall\{i, j\} \in N, \\
& M_{i j} \geq q_{j} N_{i j}, \quad \forall\{i, j\} \in N, \\
& N_{i j}=0, \quad \forall\{i, j\} \in \bar{N}_{1}, \\
& R_{i j}^{S}=R_{i j}^{C}=R_{i j}^{E}=1, \quad \forall\{i, j\} \notin \bar{N}_{2}, \\
& R_{i j}^{S}<1 \text {, } \\
& R_{i j}^{C}>1 \text {, } \\
& R_{i j}^{E}>1 \text {, } \\
& \forall\{i, j\} \in \bar{N}_{2} \text {, } \\
& \sum_{i \in M} \sum_{k \in M}\left[\frac{L_{i k}^{V}}{V_{i k}^{V}} N_{i k}+\sum_{j \in U} y_{i j k}\left(T_{i j k}-\frac{L_{i k}^{V}}{V_{i k}^{V}} N_{i k}\right)\right] \leq T^{w}, \\
& \sum_{i \in M} \sum_{k \in M}\left[\frac{L_{i k}^{V}}{R_{i k}^{S} V_{i k}^{V}} N_{i k}+\sum_{j \in U} y_{i j k}\left(T_{i j k}-\frac{L_{i k}^{V}}{R_{i k}^{S} V_{i k}^{V}} N_{i k}\right)\right] \leq T^{w}, \\
& T_{i j k} \geq 0, \quad \forall i, k \in M, \forall j \in U, \\
& W_{i j} \geq 0, \quad \forall\{i, j\} \in N, \\
& N_{i j}=\{0,1\} \text {, } \\
& N_{00}=0 \text {, } \\
& \forall\{i, j\} \in N \text {, } \\
& Z_{i j k} \in\{0,1\}, \quad \forall i, j, k \in M \text {. }
\end{aligned}
$$

Constraints (8) and (9) ensure that each customer is served by a vehicle or a UAV. Constraint (10) ensures that the numbers of vehicles leaving and entering the warehouse are the same. Constraint (11) ensures that each customer's demand is met. Constraint (12) synchronizes vehicle and UAV so that the vehicle picks up the UAV at node $k$. We will relax the restrictions (12) in Section 4 to allow vehicles to make multiple stops before receiving the UAV. Constraints
(13) and (14) are constraints on vehicle and UAV payloads, respectively. The distance range limit and the time range limit of UAV are, respectively, guaranteed by constraints (15) and (16d), where is a very large value. The flight time of $\mathrm{UAV}, T_{i j k}$, is the larger one in the travel time of UAV (16a) and vehicle under circumstance 1 (16b) or vehicle under circumstance 2 (16c). The constraint (17) determines the vehicle weight described in the preceding paragraph. Note 
that a simple lower bound (constraint (18)) can be included on the load of the vehicle at any time. Constraint (19a) ensures vehicles are forbidden to run on the edges of traffic restriction under circumstance 1 , and constraints (19b) and (19c) ensure that the average speed, variable cost, and $\mathrm{CO}_{2}$ emission of the vehicle on the edges of traffic restriction under circumstance 2 are changed. (20a) and (20b) are the constraints of working hours under circumstances 1 and 2 , respectively, where $T^{w}$ is the upper limit of working hours, normally 8 hours. $\sum_{j \in U} y_{i j k}\left(T_{i j k}-\left(L_{i k}^{V} / V_{i k}^{V}\right) N_{i k}\right)$ is the time length of the vehicle waiting for its UAV, where $y_{i j k}=1$ if $T_{i j k}>\left(L_{i k}^{V} / V_{i k}^{V}\right) N_{i k}$; otherwise, $y_{i j k}=0$. Finally, we can avoid the use of UAVs for specific routes (perhaps near airports) or for specific customers (who cannot receive such deliveries) by setting $Z_{i j k}=Z_{h i j}=0$ for route $\{i, j\}$ or $Z_{i j k}=0$ for customer j.

\section{Solution Methodology}

The application of our genetic algorithm in our routing problem with UAV is described below.

4.1. Design. In the design of our genetic algorithm, we adapt the following service strategy. The work should be completed within one day; that is, all parcels should be delivered within legal working hours, normally 8 hours. Meanwhile, fuel consumption should be minimized, or $\mathrm{CO}_{2}$ emission should be minimized. Firstly, we can run our algorithm, where only a pair of a UAV and a vehicle is used, to calculate the total working hours. Then, the number of needed vehicles is determined by dividing the total working hours by the legal working hours. After that, the region is divided according to the number of vehicles by a line (or lines) crossing the warehouse, where the number of customers served by each vehicle and the service time of each vehicle are as close to each other as possible.

In our algorithm, we first sequence the customers (please see the methods in 4.1.2). The pair of a vehicle and a UAV serves customers according to the sequence. Then, we group customers randomly as follows. Generate a random positive integer $x \in[1, n]$, where $n$ is the number of total customers served by a pair of a UAV and a vehicle. And the first $x$ customers are put into the first group. Repeat it till all customers are grouped. As a matter of course, if there are more than one edge where vehicles are forbidden, and they are divided into different groups. If there is only one customer in the group, the vehicle serves the customer. Otherwise, the UAV serves a customer randomly selected from the group, except the last one, where the UAV is retrieved, and the vehicle serves the others. If there is an edge connecting two customers in the group, where vehicles are forbidden, we randomly select one from these two customers for the UAV to serve. Based on this strategy, we developed a genetic algorithm to solve the proposed minimization problem (fuel consumption and $\mathrm{CO}_{2}$ emissions or total cost).

4.1.1. Encoding. We use natural number coding method to encode customers, warehouse, and group information. That is, we use positive integers from 1 to $n$ to encode customers and encode the warehouse as 0 . And we randomly generated positive integer(s) to encode the number of customers in a service group.

4.1.2. Initial Population Generation. In our GA, the initial population is artificially optimized to reduce iteration times and improve optimization efficiency. Specifically, we equally divide the service area into subareas of an even number. Then, we arrange the service sequence of the initial individuals as follows. The pair of a vehicle and a UAV serves customers in one of the nearest subareas from the nearest customer to the farthest customer and goes to an adjacent subarea to serve customers from the farthest to the nearest. Repeat it until all customers are served, and both the UAV and the vehicle come back into the warehouse. After that, we group the customers and obtain the initial population generation.

The following is an example to illustrate the service strategy, grouping, encoding, and initial population generation of our algorithm. There are 20 customers who should be satisfied. We encode them as $\{1,2,3,4,5,6,7,8,9,10,11$, $12,13,14,15,16,17,18,19,20\}$ and the warehouse as 0 . As only 1 pair of a UAV and a vehicle is needed, they serve the whole delivery area. Then, we divide the whole delivery area into eight subareas and arrange the service sequence of the initial individuals as follows. The pair of a UAV and a vehicle serves customers in the first subarea from left to right and goes up to the second subarea to serve customers from right to left. Then, they go left to the third subarea to serve customers from right to left and go down to the fourth subarea to serve customers from left to right. After that, they go down to the fifth subarea to serve customers from right to left and go down to the sixth subarea to serve customers from left to right. Finally, they go right to the seventh subarea to serve customers from left to right and go up to the eighth to serve customers from right to left and go back to the warehouse. Now, we obtain the service sequence codes as $\{18,1,3,7,9,19,6,12,16,8,14,2,13,15,20,11,17,4,5,10\}$

(Figure 1).

Then, we randomly generate the group codes as $\{2,5,3,4,5,1\}$. So, the customers are divided into 6 groups with encoding result $\{\{18,1\},\{3,7,9,19,6\},\{12,16,8\}$, $\{14,2,13,15\},\{20,11,17,4,5\},\{10\}\}$. As there are two customers, 18 and 1, in the first group, the UAV departs from the vehicle at the location of the warehouse to deliver parcel to customer 18 and fly to customer 1. Meanwhile, the vehicle goes to serve customer 1 and retrieve the UAV there. There are 5 customers, $3,7,9,19$, and 6 , in the second group. As customer 6 is the last one, we randomly choose 7 from 3, 7, 9, and 19 to be the customer served by the UAV. So, the UAV departs from customer 13 to serve 7 and fly to 6 , while the UAV sequentially serves customers $3,9,19$, and 6 and retrieves the UAV at 6 . There are 3 customers in the third group. We randomly choose the first customer, 12 , to be served by the UAV. As a result, the UAVs fly from 6 to 12 and to 8 , while the vehicle runs from 6 to 16 and to 8 and picks up the UAV here. For the fourth group, the UAV serves customer 14 , and the vehicle sequentially serves 2,13 , 


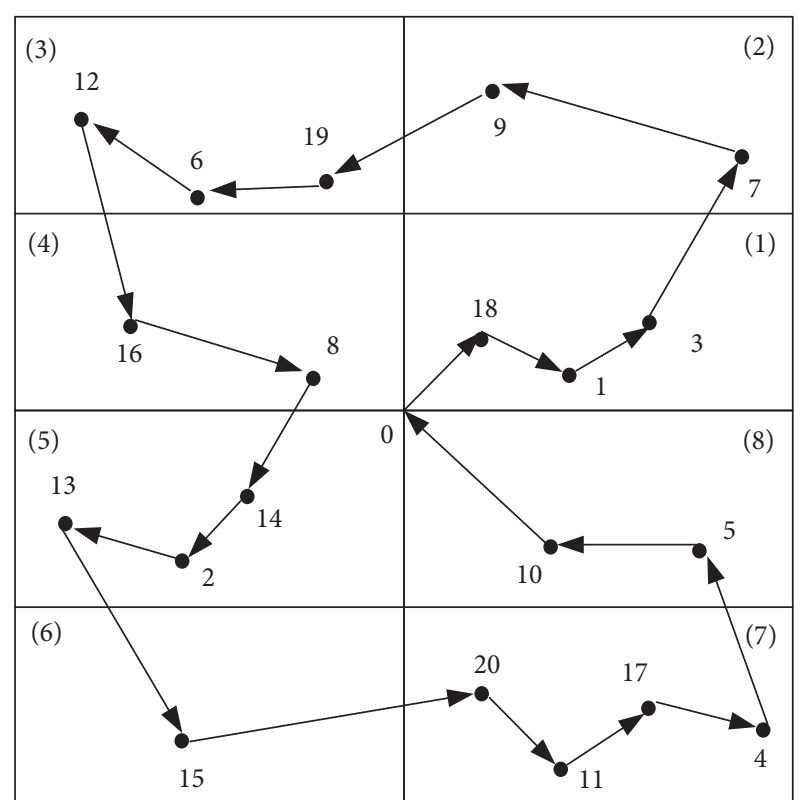

FIGURE 1: An example of partitioning service area and sequencing customers.

and 15 and retrieves the UAV here. For the fifth group, the UAV serves 4 , and the vehicle serves $20,11,17$, and 5 and retrieves the UAV here. It should be noticed that although there is only customer 10 in the last group, the UAV should depart from customer 5 to serve customer 10 and fly back to the warehouse, and the vehicle should directly run from customer 10 back to the warehouse. The initial population generation is shown in Figure 2.

\subsubsection{The Algorithms}

(1) Fitness Function. In Section 3, we explained the design of the model in detail, in which the objective function mentioned mainly includes the load of the vehicle and the UAV, the distance between customers and the corresponding energy consumption function and cost function. Accordingly, the fitness function can be defined as fitness $(X)=(1 / T(X))$, where $X$ is the combination of service sequence and grouping and $T(X)$ is the target function.

(2) Selection, Crossover, and Variation. In the process of optimal individual selection, the greater the fitness of an individual, the greater the probability of being selected as the parent of the next generation, and the method is similar to roulette. As long as the fitness of an individual is large enough, the same individual in the genetic algorithm can exist in different generations. In the specific selection, select the elite individual retention strategy and copy the individuals with the highest fitness to the alternative parent group of cross-matching, then cross match all the individuals in the parent group, select the individuals with the highest fitness, and repeat this process, so as to gradually eliminate the individuals with poor fitness, leaving the elite individuals. At the same time, the method of double point crossing and real-value variation is adopted.

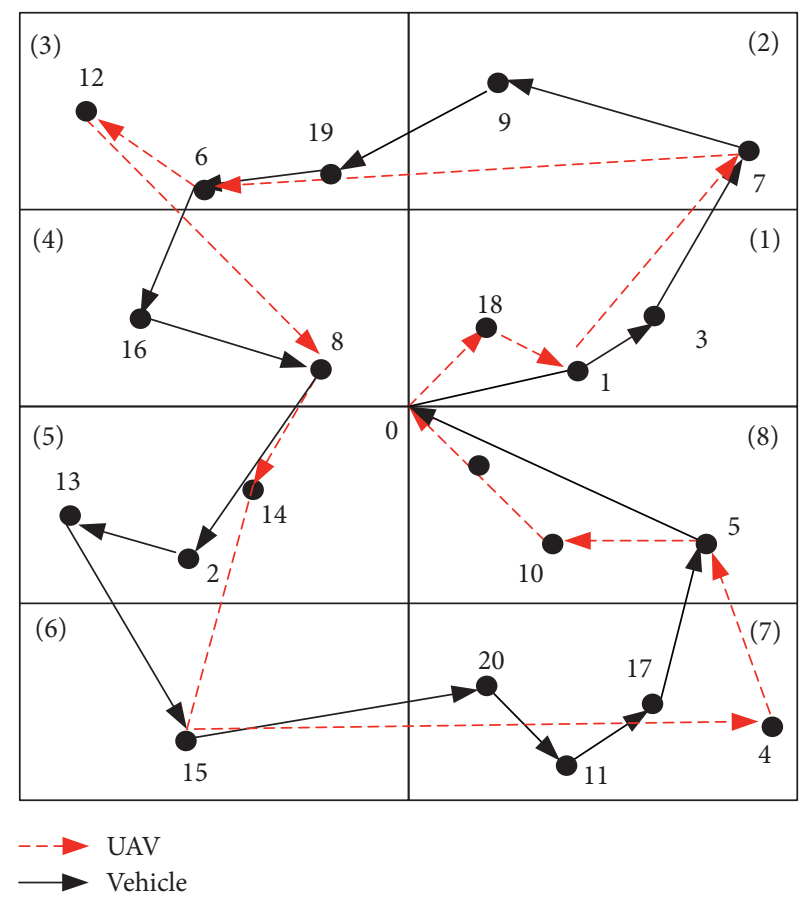

FIgURE 2: An example of an initial population generation.

(3) Termination Conditions. After several iterations, when the output iteration results meet the following conditions, the convergence value is reached and the algorithm is terminated:

(1) There was no significant change in the fitness value after continuous iteration; in other words, the change is less than $1 \%$ of the fitness value

(2) The population no longer evolves and the number of iterations reaches the set value

4.2. Algorithm Steps. In this paper, the algorithm steps are as follows:

(1) Determine the coding mechanism, generate the initial population, and set the probability of cross variation and the maximum number of iterations.

(2) Evaluate the fitness of the current population and find the chromosome with the minimum objective function value and corresponding function value.

(3) Determine whether the termination conditions are met. Output the optimal chromosome and corresponding solutions if satisfied; otherwise, select the current population again.

(4) Repeated crossover and mutation in the newly obtained generation.

(5) Evaluate the fitness of the results and move to (3).

\section{Experimentation}

In this section, we use the software Matlab R2017a to carry out the experiment and test our model and GA. The computer was used in the experiment is ASUS Rock 5 
Generation, whose configuration is $\operatorname{Inter}(\mathrm{R}) \operatorname{Core}(\mathrm{TM})$ i7$8550 \mathrm{u}$ processor $(1.8 \mathrm{GHz}$ base, $1.99 \mathrm{GHz}$ Max Turbo), 128 GB SSD, 8 GB RAM.

5.1. Data Parameters. The range of customer demand, $q_{j}$, is $1 \sim 10$ pounds, among which $80 \%$ is no more than 5 pounds, and the position of each customer $(x, y)$ coordinate values $x$ and $y$ randomly evenly generated in the interval $[-10,+10]$. The distance of the vehicle from customer $i$ to $j$ is $L_{i j}^{V}$, following the Manhattan metric; the distance of the UAV from customer $i$ to $j$ is $L_{i j}^{U}$, following the Euclidean metric; in addition, the warehouse is located at the center of the region $(0,0)$.

Referring to Chiang et al. [2], we set the other parameters as follows.

The empty weight of the UAV, $W^{U}$, is 55 pounds; the payload of the UAV, $M^{U}$, is 5 pounds; the maximum flight distance of the UAV, $L^{U}$, is 10 miles; and the average flight speed of the UAV from customer $i$ to $j, V_{i j}^{U}$, is 25 miles per hour. For roads with the second kind of traffic restriction, $R_{i j}^{C}=R_{i j}^{E}=1.2$ and $R_{i j}^{S}=0.8333$.

The empty weight of the vehicle (truck), $W^{V}$, is 6100 pounds, its payload, $M^{V}$, is 6000 pounds, and the average speed of the vehicle from customer $i$ to $j, V_{i j}^{V}$, is 25 miles per hour.

The fixed cost of a pair of a vehicle and a UAV, $C^{F}$, is $\$ 500$, the unit route cost of a vehicle, $C^{V}$, is $\$ 0.00016 /$ poundmile, and the unit route cost of a UAV, $C^{U}$, is $\$ 0.00036364 /$ pound-mile.

Referring to Goodchild and Toy [33], the weighted average emission of vehicles, WAER, is $1.2603 \mathrm{Kg} /$ pound-mile, the $\mathrm{CO}_{2}$ emission from the power generation facilities in the generation of electricity, PGFER, is $3.773 \times 10^{-4} \mathrm{Kg} / \mathrm{Wh}$, and the average energy requirement of $\mathrm{UAV}, \mathrm{AER}$, is $3.3333 \mathrm{Wh} /$ pound-mile.

5.2. The Examples. Firstly, we present an example of 20 customers under the two separate kinds of traffic restrictions, in order to analyze the efficiency of UAV delivery overcoming traffic restrictions. When there are 20 customers, only one vehicle or a pair of a vehicle and a UAV is needed. Figure 3(a) shows the routing of a vehicle under no traffic restriction, and Figures 3(b)-3(d) separately show the routings of a vehicle and a pair of a vehicle and a UAV under two kinds of traffic restrictions. From Figure 3(d), we can find that the routings under two kinds of traffic restrictions are the same.

The results of costs and emissions under different circumstances are as follows. When a vehicle is under no traffic restriction, the $\mathrm{CO}_{2}$ emission is $161.3032 \mathrm{~kg}$, and the variable cost is $\$ 134.09$. When a vehicle is under the first kind of traffic restriction, the $\mathrm{CO}_{2}$ emission is $167.4327 \mathrm{Kg}$, and the variable cost is $\$ 139.19$. When a vehicle is under the second kind of traffic restriction, the $\mathrm{CO}_{2}$ emission is $166.3727 \mathrm{Kg}$, and the variable cost is $\$ 138.30$. When a pair of a vehicle is under both the first and the second kinds of traffic restriction, the $\mathrm{CO}_{2}$ emissions are the same, which is
$151.6250 \mathrm{~kg}$, and the variable cost is the same too, which is $\$ 113.97$.

From Figure 3(d) and the result of costs and emissions, we can find that the routing as well as cost and emission under the first kind of traffic restriction are the same as those under the second. The main reason is no matter which kind of the traffic restriction is, we always use a UAV instead of a vehicle to deliver a parcel when facing a traffic restriction.

Comparing Figures $3(\mathrm{a})-3(\mathrm{~d})$, as well as the corresponding result of costs and emissions, we can obtain the following conclusions.

Firstly, traffic restrictions raise the cost and emission, and first kind of traffic restriction raises them further higher. The main reason is that when a road in the routing is blocked for the delivery vehicle, it has to choose other road, while if there is only one lane available, the vehicle could still run through the road if the cost and emission are lower than those running through other roads. Therefore, the cost and emission under the second kinds of traffic restriction are higher than those under no traffic restriction but lower than those under the first kind of traffic restriction.

Secondly, UAV delivery can always reduce the cost and emission no matter if there is traffic restriction and which kind of traffic restriction is. Furthermore, UAV delivery saves more cost and cuts more emission under the first kind of traffic restriction than that under the second, as the cost and emission under the first kind of traffic restriction are the highest and those under two kinds of traffic restrictions are the same.

In the following section, firstly, we present the result of 200 customers under the first kind of traffic restriction. The location of the 200 customers and the weight of their demand of were randomly generated and shown in Table 1. 2 cars are needed for customer service within 8 hours per day. The service times of each vehicle are 6.65 hours and 6.94 hours, respectively. The routing of the two pairs of vehicles and drones is shown in Figure 4, and the results of costs and emissions are as follows.

The $\mathrm{CO}_{2}$ emission of the first vehicle is $209.5249 \mathrm{Kg}$, and that of the corresponding $\mathrm{UAV}$ is $0.1277 \mathrm{Kg}$. The $\mathrm{CO}_{2}$ emission of the second vehicle is $218.6621 \mathrm{~kg}$ and that of the corresponding UAV is $0.1636 \mathrm{~kg}$. Therefore, the total $\mathrm{CO}_{2}$ emission from the package delivery is $428.4783 \mathrm{~kg}$. If no $\mathrm{UAV}$ is used, the total $\mathrm{CO}_{2}$ emission will be $467.8864 \mathrm{~kg}$.

The variable costs of the first pair of a vehicle and a UAV are $\$ 145.52$ and $\$ 2.03$, respectively, and the variable costs of the second pair of a vehicle and a UAV are $\$ 152.21$ and $\$ 2.60$. Therefore, the total cost of serving the 200 customers is $\$ 1302.36$. The total cost will be $\$ 1388.95$, if no UAV is used.

5.3. Extended Examples. In the section, we extend our example to 300 and 400 customers.

5.3.1. The Impact of UAVs on $\mathrm{CO}_{2}$ Emissions. Table 2 shows the $\mathrm{CO}_{2}$ emissions without the use of UAVs, and Table 3 shows the $\mathrm{CO}_{2}$ emissions with the use of UAVs. Table 4 


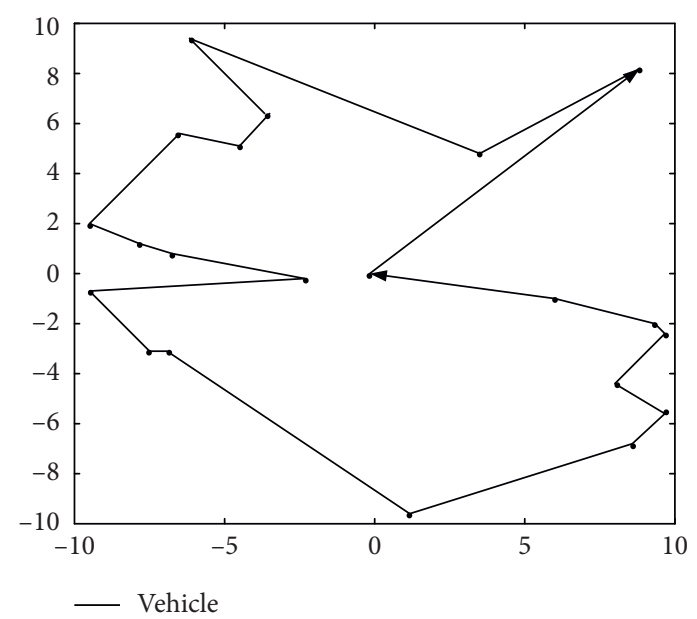

(a)

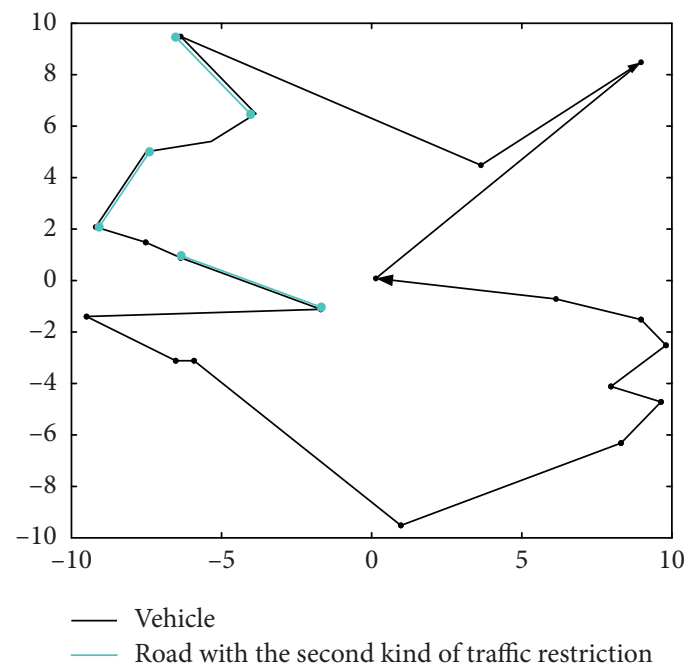

(c)

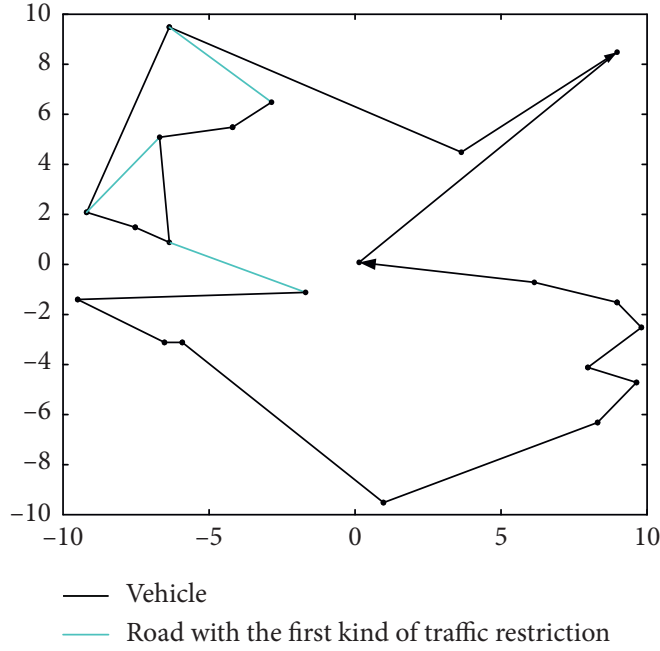

(b)

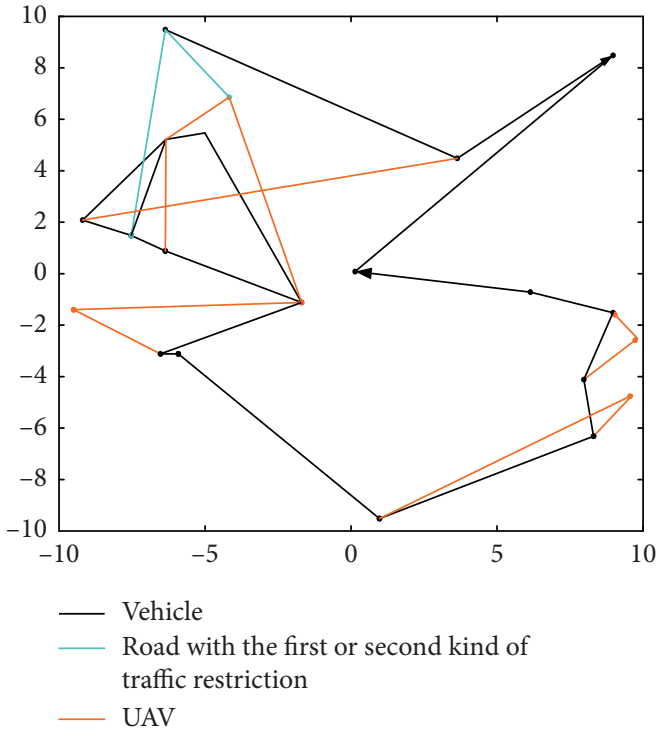

(d)

Figure 3: Routings under different circumstances: (a) routing of only vehicle under no traffic restriction; (b) routing of only vehicle under the first kind of traffic restriction; (c) routing of only vehicle under the second kind of traffic restriction; (d) routing of vehicle with UAV under two kinds of traffic restrictions.

shows the intuitive comparison between the results without UAVs and those with UAVs.

It can be seen from Tables $2-4$ that, in the case of traffic restrictions, with the increase in the number of customers, the number of vehicles and $\mathrm{CO}_{2}$ emissions are also rising. From Table 4, we can find that when there are 300 or 400 customers, 3 and 4 vehicles are needed, respectively, when no UAVs are being used, while only 2 and 3 vehicles are needed, respectively, when UAVs are being used. We also found that using UAVs can effectively reduce $\mathrm{CO}_{2}$ emission even when there are 200 customers and the number of vehicles is not reduced.

5.3.2. The Impact of UAVs on Costs. Now, we analyze the impact of using UAVs on delivery costs. Table 5 shows the delivery costs without UAV, Table 6 shows the delivery costs with UAV, and Table 7 shows the comparison between the costs without UAVs and that with UAVs.

From Tables 5-7, we found that the variable cost, fixed cost, and total cost rise with the increase in the number of customers. And as per our expectation, we found from Table 7 that using UAVs can always effectively reduce variable cost and total cost, as well as fixed cost when there are 300 and 400 customers because the number of required vehicles is reduced. Our expectation is that using UAVs greatly reduces the variable cost by at least $28.64 \%$ and up to $43.47 \%$.

Tables 4 and 7 show that using UAVs in package delivery reduces not only the variable cost and total costs but also $\mathrm{CO}_{2}$ emission. In other words, UAV delivery is environmentally and economically beneficial. 
TABLE 1: Location of the 200 customers and the weight of their demand.

\begin{tabular}{|c|c|c|c|c|c|}
\hline Customer & Location & Weight of demand (pound) & Customer & Location & Weight of demand (pound) \\
\hline 1 & $(3.5985,3.6248)$ & 2.6464 & 101 & $(6.6985,-7.8311)$ & 2.4695 \\
\hline 2 & $(1.6070,2.0104)$ & 2.0035 & 102 & $(-1.9531,-3.3253)$ & 4.6376 \\
\hline 3 & $(6.7836,3.9658)$ & 4.9405 & 103 & $(-7.8722,-1.6863)$ & 5.3956 \\
\hline 4 & $(2.7794,2.7118)$ & 0.4074 & 104 & $(5.0983,-0.4058)$ & 5.5206 \\
\hline 5 & $(5.4746,-1.4383)$ & 5.5835 & 105 & $(-7.3811,-9.4588)$ & 2.7891 \\
\hline 6 & $(3.7286,-5.7173)$ & 2.6218 & 106 & $(-9.7011,-9.1042)$ & 0.6035 \\
\hline 7 & $(-3.9446,-0.7422)$ & 0.9957 & 107 & $(-8.0567,4.1457)$ & 5.7241 \\
\hline 8 & $(-6.6992,6.3058)$ & 0.8271 & 108 & $(-6.8909,-9.5609)$ & 2.0171 \\
\hline 9 & $(-8.7527,-4.0516)$ & 0.7294 & 109 & $(-5.7863,4.4279)$ & 6.1105 \\
\hline 10 & $(-4.4899,-5.3138)$ & 1.3197 & 110 & $(7.3203,5.0068)$ & 2.3235 \\
\hline 11 & $(4.4820,-3.0529)$ & 6.0178 & 111 & $(8.0247,-7.8543)$ & 4.213 \\
\hline 12 & $(1.4923,-1.8090)$ & 4.5951 & 112 & $(-5.0841,1.2691)$ & 5.5378 \\
\hline 13 & $(-8.3485,2.9984)$ & 3.7066 & 113 & $(3.0984,-5.5088)$ & 4.0103 \\
\hline 14 & $(3.5833,-0.3746)$ & 4.2485 & 114 & $(-8.4013,-2.8959)$ & 3.9066 \\
\hline 15 & $(-8.8362,3.5809)$ & 4.8984 & 115 & $(-1.5125,3.4101)$ & 4.5946 \\
\hline 16 & $(-3.4012,2.5994)$ & 2.6331 & 116 & $(4.7787,8.0351)$ & 2.5481 \\
\hline 17 & $(-3.2365,0.3061)$ & 5.1189 & 117 & $(0.5036,-1.8153)$ & 4.4859 \\
\hline 18 & $(-9.3533,3.8708)$ & 2.3671 & 118 & $(9.2231,-5.6816)$ & 6.1265 \\
\hline 19 & $(5.7948,3.0688)$ & 1.6931 & 119 & $(-0.7709,-0.2432)$ & 0.8386 \\
\hline 20 & $(-9.3260,2.3723)$ & 6.0346 & 120 & $(0.1749,0.4201)$ & 2.5176 \\
\hline 21 & $(5.5314,-8.4922)$ & 3.9256 & 121 & $(6.5870,-5.7042)$ & 3.4506 \\
\hline 22 & $(-5.3389,-1.8910)$ & 4.0786 & 122 & $(5.1065,-4.8937)$ & 5.6184 \\
\hline 23 & $(5.0150,1.0702)$ & 0.5409 & 123 & $(-6.5286,-4.1542)$ & 4.5411 \\
\hline 24 & $(5.3291,8.8109)$ & 0.726 & 124 & $(4.8201,0.3814)$ & 1.8215 \\
\hline 25 & $(5.1424,2.8790)$ & 3.9069 & 125 & $(-8.0454,2.6018)$ & 3.8553 \\
\hline 26 & $(-8.5682,-4.6079)$ & 0.2204 & 126 & $(5.8765,0.1324)$ & 0.413 \\
\hline 27 & $(2.4282,-7.1526)$ & 5.7625 & 127 & $(-8.9043,2.6839)$ & 2.5147 \\
\hline 28 & $(5.3858,3.9510)$ & 5.8148 & 128 & $(3.2194,4.7627)$ & 3.5148 \\
\hline 29 & $(-3.1541,-4.4266)$ & 6.0896 & 129 & $(-3.9258,0.0123)$ & 4.7808 \\
\hline 30 & $(6.9408,0.1415)$ & 1.0124 & 130 & $(0.0193,9.3367)$ & 4.6436 \\
\hline 31 & $(6.9864,6.0812)$ & 2.2663 & 131 & $(-1.7297,-2.4018)$ & 5.7673 \\
\hline 32 & $(-2.2162,-7.0139)$ & 2.0512 & 132 & $(7.9912,3.1555)$ & 4.0447 \\
\hline 33 & $(1.7152,6.3001)$ & 1.4065 & 133 & $(-1.3450,1.1250)$ & 2.9504 \\
\hline 34 & $(7.2730,1.6194)$ & 0.4455 & 134 & $(4.6406,2.6336)$ & 6.0704 \\
\hline 35 & $(-9.0451,-1.9021)$ & 5.6629 & 135 & $(-1.8672,-3.1354)$ & 3.7547 \\
\hline 36 & $(3.9235,3.1469)$ & 5.9208 & 136 & $(4.4430,7.4150)$ & 0.8491 \\
\hline 37 & $(-4.1134,9.0178)$ & 0.7815 & 137 & $(-7.3532,3.6512)$ & 0.8283 \\
\hline 38 & $(4.1405,0.6544)$ & 5.1735 & 138 & $(6.8433,5.5395)$ & 2.5081 \\
\hline 39 & $(-9.0047,2.8633)$ & 5.2433 & 139 & $(8.1880,8.9098)$ & 2.8487 \\
\hline 40 & $(3.9065,-5.4808)$ & 3.8613 & 140 & $(-8.6609,-7.1078)$ & 4.798 \\
\hline 41 & $(6.8066,6.7106)$ & 1.008 & 141 & $(6.5072,1.6621)$ & 4.9815 \\
\hline 42 & $(1.4364,-6.4336)$ & 4.315 & 142 & $(-1.7420,8.7680)$ & 1.4472 \\
\hline 43 & $(-8.9608,-7.4226)$ & 5.8182 & 143 & $(-8.7449,-4.7519)$ & 3.4111 \\
\hline 44 & $(0.6380,1.0638)$ & 5.9632 & 144 & $(2.5800,9.5467)$ & 5.5106 \\
\hline 45 & $(-5.3386,-5.5256)$ & 1.804 & 145 & $(-6.2282,2.4928)$ & 3.4125 \\
\hline 46 & $(-2.7935,0.2916)$ & 3.8737 & 146 & $(9.7491,2.2369)$ & 3.6908 \\
\hline 47 & $(-3.2275,-7.6894)$ & 6.1055 & 147 & $(5.0762,-8.1123)$ & 3.9738 \\
\hline 48 & $(6.0844,5.0847)$ & 6.0703 & 148 & $(-2.1303,7.2874)$ & 2.6361 \\
\hline 49 & $(-5.1356,-5.9556)$ & 3.0152 & 149 & $(-6.2300,-6.5167)$ & 2.699 \\
\hline 50 & $(2.6515,7.7721)$ & 0.0072 & 150 & $(6.2027,-0.4723)$ & 4.6166 \\
\hline 51 & $(-0.0056,-0.1799)$ & 3.3836 & 151 & $(2.0571,3.5960)$ & 5.8956 \\
\hline 52 & $(2.7594,-4.9004)$ & 5.8689 & 152 & $(9.7581,5.6005)$ & 1.1632 \\
\hline 53 & $(6.6679,7.1263)$ & 5.246 & 153 & $(5.2829,1.7994)$ & 2.5925 \\
\hline 54 & $(9.8432,3.9686)$ & 1.3284 & 154 & $(-2.2265,6.9336)$ & 2.3741 \\
\hline 55 & $(-5.3313,6.4587)$ & 1.3662 & 155 & $(-8.9635,6.1876)$ & 3.3864 \\
\hline 56 & $(-7.3582,2.8845)$ & 5.5513 & 156 & $(7.3399,-1.8563)$ & 2.5694 \\
\hline 57 & $(0.1812,2.1129)$ & 3.2123 & 157 & $(-3.4740,-8.4158)$ & 5.951 \\
\hline 58 & $(-7.1705,7.0471)$ & 2.6717 & 158 & $(-0.1858,1.4740)$ & 0.9742 \\
\hline 59 & $(8.5137,5.5096)$ & 4.5286 & 159 & $(-0.3142,-5.9115)$ & 2.0432 \\
\hline 60 & $(-1.5415,-7.7999)$ & 3.7548 & 160 & $(-8.6928,3.0577)$ & 4.5069 \\
\hline
\end{tabular}


TABLE 1: Continued.

\begin{tabular}{|c|c|c|c|c|c|}
\hline Customer & Location & Weight of demand (pound) & Customer & Location & Weight of demand (pound) \\
\hline 61 & $(6.4396,6.4118)$ & 3.0396 & 161 & $(-8.9804,1.7362)$ & 3.4904 \\
\hline 62 & $(8.1804,-3.8801)$ & 5.6087 & 162 & $(0.8940,8.4429)$ & 1.8262 \\
\hline 63 & $(-9.6267,6.2938)$ & 1.5951 & 163 & $(-1.6117,-7.0373)$ & 2.0569 \\
\hline 64 & $(9.7571,7.7541)$ & 5.2335 & 164 & $(-6.0486,-4.1884)$ & 0.8061 \\
\hline 65 & $(-7.4305,-1.9970)$ & 6.0315 & 165 & $(0.5796,-2.2476)$ & 4.9394 \\
\hline 66 & $(-4.1666,9.7131)$ & 4.442 & 166 & $(-3.0904,-9.0519)$ & 1.6785 \\
\hline 67 & $(8.6054,-5.4922)$ & 5.3479 & 167 & $(8.5203,-7.3574)$ & 2.4377 \\
\hline 68 & $(-9.7305,1.9685)$ & 3.6987 & 168 & $(9.3078,-5.7472)$ & 2.9458 \\
\hline 69 & $(4.0727,-8.2039)$ & 3.3089 & 169 & $(-1.0789,-5.0646)$ & 6.195 \\
\hline 70 & $(-3.8506,8.7982)$ & 3.216 & 170 & $(3.4054,-9.4300)$ & 0.0608 \\
\hline 71 & $(-7.1437,7.1571)$ & 2.9886 & 171 & $(-8.1767,-0.5804)$ & 2.5696 \\
\hline 72 & $(8.6707,3.3893)$ & 4.1855 & 172 & $(1.0544,-1.7339)$ & 4.0753 \\
\hline 73 & $(-6.2558,-1.5341)$ & 3.2457 & 173 & $(-8.5217,-1.5462)$ & 0.9069 \\
\hline 74 & $(-4.9621,-2.4825)$ & 1.0398 & 174 & $(7.3960,5.3860)$ & 2.8008 \\
\hline 75 & $(6.5565,-1.0670)$ & 1.6786 & 175 & $(7.8358,-1.8235)$ & 4.3691 \\
\hline 76 & $(-9.1507,-8.5815)$ & 2.1336 & 176 & $(0.7854,-0.1814)$ & 2.1629 \\
\hline 77 & $(2.3058,3.3969)$ & 3.1581 & 177 & $(7.3398,4.4719)$ & 4.8862 \\
\hline 78 & $(-6.1230,1.4946)$ & 1.635 & 178 & $(3.6908,0.0740)$ & 5.8748 \\
\hline 79 & $(-6.8841,5.0681)$ & 2.3038 & 179 & $(-1.7045,5.2675)$ & 3.5177 \\
\hline 80 & $(3.1371,-3.3084)$ & 5.5688 & 180 & $(-4.3713,-5.1950)$ & 3.6966 \\
\hline 81 & $(1.7032,-5.9946)$ & 0.4024 & 181 & $(8.7083,-8.4181)$ & 2.1445 \\
\hline 82 & $(-1.5590,5.8996)$ & 4.9225 & 182 & $(3.5814,9.0920)$ & 4.3845 \\
\hline 83 & $(-5.1026,7.0859)$ & 3.2166 & 183 & $(4.9116,-6.6143)$ & 2.1252 \\
\hline 84 & $(-2.3133,-0.0468)$ & 1.2746 & 184 & $(-9.3055,-6.6730)$ & 5.3135 \\
\hline 85 & $(-1.9291,-8.8507)$ & 5.5929 & 185 & $(1.1326,9.1880)$ & 2.7653 \\
\hline 86 & $(4.6261,-7.2169)$ & 0.2662 & 186 & $(2.4269,9.3005)$ & 3.8122 \\
\hline 87 & $(7.3182,2.3801)$ & 0.2147 & 187 & $(-8.2876,9.1493)$ & 4.9769 \\
\hline 88 & $(6.0759,-3.9352)$ & 5.8295 & 188 & $(-9.5075,5.6753)$ & 2.5376 \\
\hline 89 & $(8.0556,-4.2223)$ & 2.5624 & 189 & $(8.3507,-0.2268)$ & 6.1962 \\
\hline 90 & $(-5.7516,8.0645)$ & 5.6345 & 190 & $(6.5826,3.3623)$ & 3.7944 \\
\hline 91 & $(8.1302,8.2009)$ & 3.6932 & 191 & $(7.2440,-6.8963)$ & 3.5641 \\
\hline 92 & $(3.9469,4.9619)$ & 1.1317 & 192 & $(-1.3492,-6.6058)$ & 4.005 \\
\hline 93 & $(2.0032,-8.4006)$ & 1.2813 & 193 & $(1.2460,-5.5328)$ & 0.036 \\
\hline 94 & $(7.8395,7.5786)$ & 1.4329 & 194 & $(-1.1003,0.0472)$ & 5.9488 \\
\hline 95 & $(-7.5101,-8.0132)$ & 0.9963 & 195 & $(-2.2261,-3.0986)$ & 1.1039 \\
\hline 96 & $(8.9171,7.1257)$ & 5.1115 & 196 & $(2.2383,-1.0874)$ & 1.6342 \\
\hline 97 & $(-0.0308,-6.0585)$ & 2.2 & 197 & $(0.0418,-8.8158)$ & 5.8702 \\
\hline 98 & $(4.7966,9.9410)$ & 4.871 & 198 & $(3.8139,2.0351)$ & 4.0963 \\
\hline 99 & $(-9.4510,-7.4800)$ & 0.9984 & 199 & $(5.2971,-5.0442)$ & 4.8425 \\
\hline 100 & $(6.4788,-0.9906)$ & 1.6839 & 200 & $(5.0138,-0.5799)$ & 4.0817 \\
\hline
\end{tabular}

\section{Discussion}

In this paper, we classified traffic restrictions into two circumstances and studied the environmental and economic impact of UAV delivery under traffic restriction, and we found that UAV delivery can effectively save energy and cost. In other words, UAV delivery economically benefits not only logistics firms but also the sustainability of environmental development, which is supported by Chiang et al. [2]. We also found that, under the two kinds of traffic restrictions, UAV delivery can accomplish deliveries that cannot be carried out or are carried out at much higher costs by vehicles only, and it is more effective under the first kind of traffic restriction, which has not been studied.

We would like to point out that, with the development of UAV technology, UAV delivery is more and more popularly accepted as the best resolution of "last-mile" delivery problem. Amazon, UPS, Walmart, Google, JD.com, and
Alibaba started to apply UAVs in package delivery. In this paper, we proposed managers an effective model to create competitiveness by optimally coordinating vehicles and UAVs. Shifting small package delivery from trucks to UAVs greatly reduces energy consumption and costs in package delivery. As a matter of course, to realize the environmental and economic benefits of UAV delivery, firms should carefully plan and control the routing and cooperation of vehicles and UAVs. Operational decisions can effectively reduce delivery costs and $\mathrm{CO}_{2}$ emission, as well as improve the environment [34].

Absolutely, the current power technology of UAVs limits the range and payload capacity of UAVs. At present, the battery used in UAVs is lithium ion battery with an energy density of around $300 \mathrm{Wh} / \mathrm{kg}$, while the fluorinated battery can reach $2585 \mathrm{Wh} / \mathrm{kg}$, which can greatly improve the performance of UAVs [35]. This means future developments in emerging energy sources will ease current restrictions on 


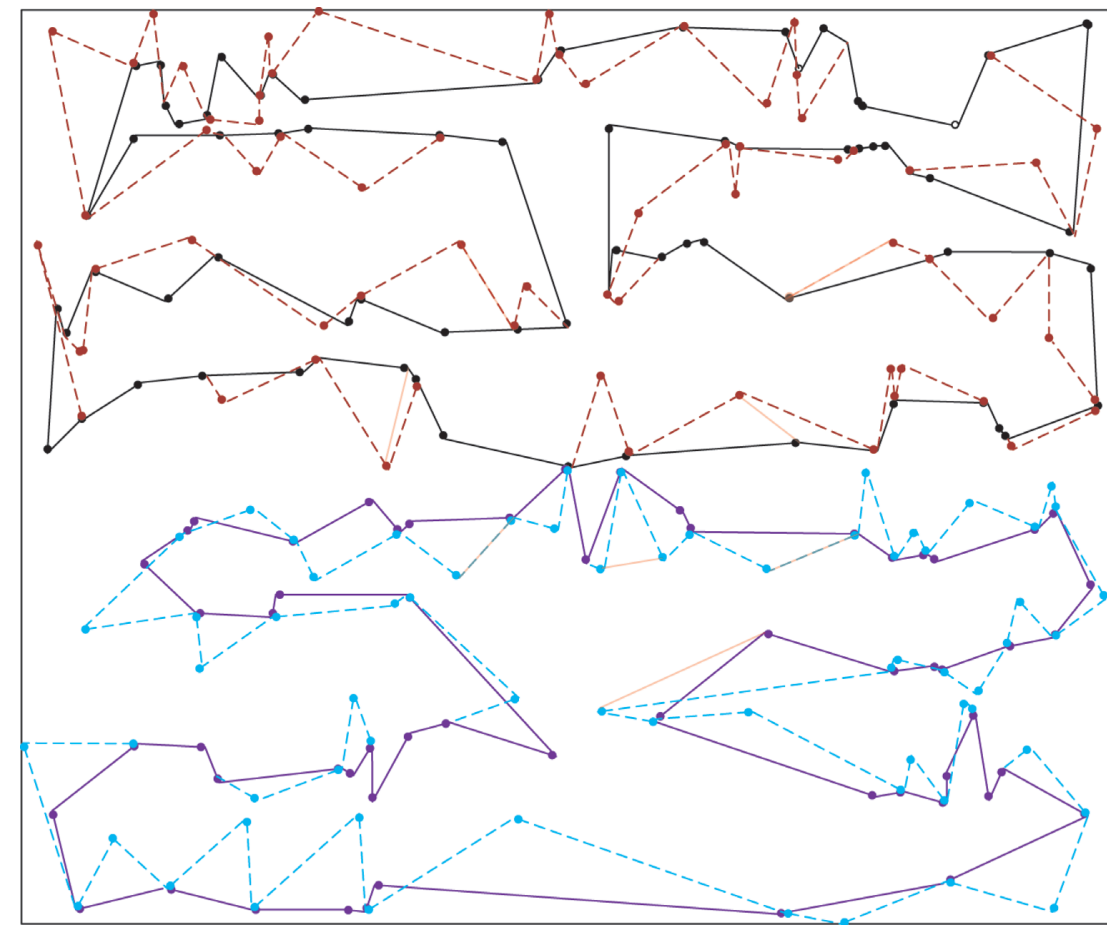
— First vehicle
— Second vehicle
- - - First UAV
- - Second UAV
Edge with traffic restriction

Figure 4: Routing of the 2 pairs of vehicles and UAVs.

TABLE 2: $\mathrm{CO}_{2}$ emissions without UAVs.

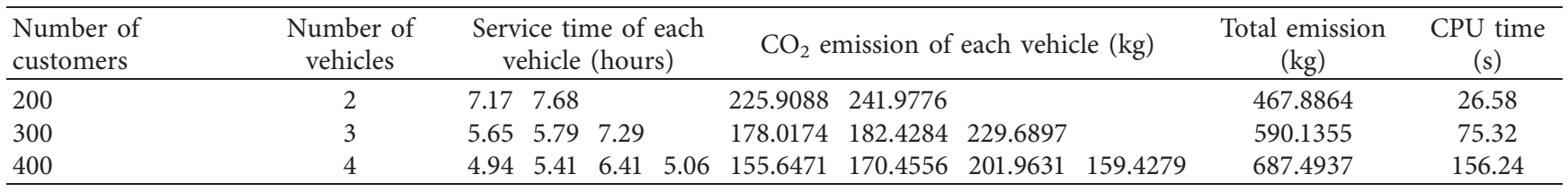

TABle 3: $\mathrm{CO}_{2}$ emissions with UAVs.

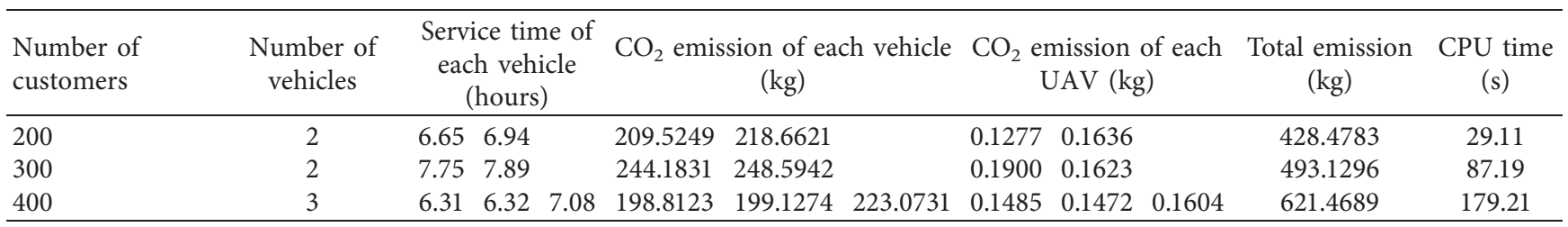

TABLE 4: Comparison of $\mathrm{CO}_{2}$ emissions of using UAVs and not using UAVs.

\begin{tabular}{|c|c|c|c|c|c|c|c|c|c|}
\hline \multirow{3}{*}{$\begin{array}{l}\text { Number of } \\
\text { customers }\end{array}$} & \multicolumn{2}{|c|}{ Number of vehicles } & \multirow{3}{*}{$\begin{array}{c}\text { Number of reduced } \\
\text { vehicles }\end{array}$} & \multicolumn{4}{|c|}{$\mathrm{CO}_{2}$ emission $(\mathrm{kg})$} & \multicolumn{2}{|c|}{$\begin{array}{l}\mathrm{CO}_{2} \text { emission } \\
\text { reduction }\end{array}$} \\
\hline & \multirow{2}{*}{$\begin{array}{l}\text { Without } \\
\text { UAV }\end{array}$} & \multirow{2}{*}{$\begin{array}{l}\text { With } \\
\text { UAV }\end{array}$} & & \multirow{2}{*}{$\begin{array}{l}\text { Without } \\
\text { UAV }\end{array}$} & \multicolumn{3}{|c|}{ With UAV } & \multirow{2}{*}{ (kg) } & \multirow{2}{*}{ (\%) } \\
\hline & & & & & Vehicle & UAV & Total & & \\
\hline 200 & 2 & 2 & 0 & 467.8864 & 428.1870 & 0.2913 & 428.4783 & 39.4081 & 9.20 \\
\hline 300 & 3 & 2 & 1 & 590.1355 & 492.7773 & 0.3523 & 493.1296 & 97.0059 & 19.67 \\
\hline 400 & 4 & 3 & 1 & 687.4937 & 621.0128 & 0.4561 & 621.4689 & 66.0248 & 10.62 \\
\hline
\end{tabular}


TABLE 5: Variable costs without UAVs.

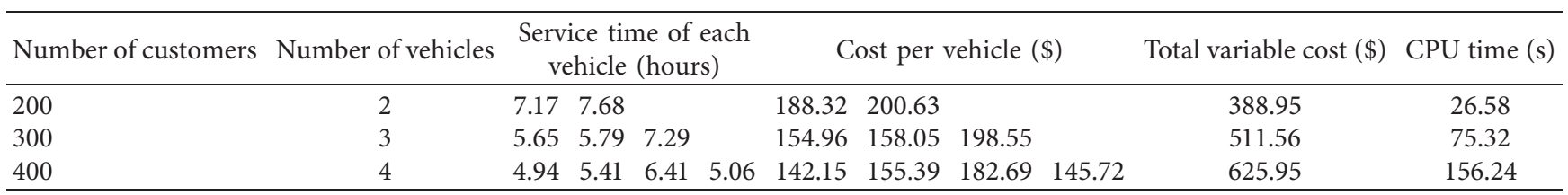

TABLE 6: Variable costs with UAVs.

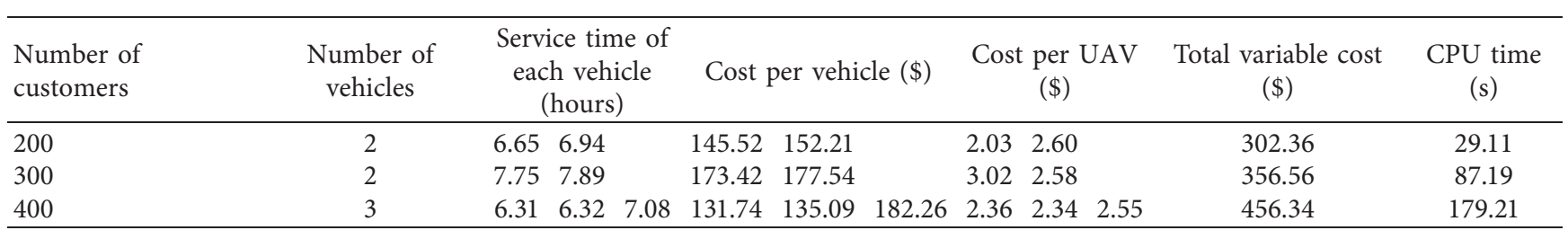

TABle 7: Comparison of the costs of using UAV and not using UAV.

\begin{tabular}{|c|c|c|c|c|c|c|c|c|c|}
\hline \multirow{2}{*}{$\begin{array}{l}\text { Number of } \\
\text { customers }\end{array}$} & \multicolumn{2}{|c|}{$\begin{array}{l}\text { Number of } \\
\text { reduced vehicles }\end{array}$} & \multirow{2}{*}{$\begin{array}{l}\text { Number of } \\
\text { reduced } \\
\text { vehicles }\end{array}$} & \multicolumn{2}{|c|}{$\begin{array}{c}\text { Number of reduced } \\
\text { vehicles }\end{array}$} & \multirow{2}{*}{$\begin{array}{c}\text { Percentage of } \\
\text { variable cost } \\
\text { reduction by using } \\
\text { UAV }(\%)\end{array}$} & \multicolumn{2}{|c|}{$\begin{array}{c}\text { Number of reduced } \\
\text { vehicles }\end{array}$} & \multirow{2}{*}{$\begin{array}{l}\text { Percentage of total } \\
\text { cost reduction by } \\
\text { using UAV (\%) }\end{array}$} \\
\hline & $\begin{array}{l}\text { Without } \\
\text { UAV }\end{array}$ & $\begin{array}{l}\text { With } \\
\text { UAV }\end{array}$ & & $\begin{array}{l}\text { Without } \\
\text { UAV }\end{array}$ & $\begin{array}{l}\text { With } \\
\text { UAV }\end{array}$ & & $\begin{array}{l}\text { Without } \\
\text { UAV }\end{array}$ & $\begin{array}{l}\text { With } \\
\text { UAV }\end{array}$ & \\
\hline 200 & 2 & 2 & 0 & 388.95 & 302.36 & 28 & 1388. & 13 & \\
\hline 300 & 3 & 2 & 1 & 511.56 & 356.56 & 43.47 & 2011.56 & 1356 & 48.28 \\
\hline 400 & 4 & 3 & 1 & 625.95 & 456.34 & 43.01 & 2625.95 & 1956.34 & 34.23 \\
\hline
\end{tabular}

drone use and may provide additional cost savings that will allow UAV-vehicle delivery of last-mile packages to be further improved.

\section{Conclusions}

In this paper, we classified traffic restrictions into two circumstances and proposed a mixed-integer (0-1 linear) UAV-vehicle routing model with these two kinds of traffic restrictions to exploit the environmental aspects of the use of UAVs in logistics. A genetic algorithm was proposed to solve the model, and an experimental analysis was made to illustrate and validate our model and the algorithm. We found that, under the two kinds of traffic restrictions, delivering with UAVs can accomplish deliveries that cannot be carried out or are carried out at much higher prices by vehicles only and can effectively save costs and reduce $\mathrm{CO}_{2}$ emissions, which is environmentally friendly. Furthermore, UAV delivery saves more cost and cuts more $\mathrm{CO}_{2}$ emission under the first kind of traffic restriction than that under the second.

\section{Data Availability}

The data used to support the findings of this study are included within the article.

\section{Conflicts of Interest}

The authors declare that there are no conflicts of interest.

\section{Acknowledgments}

This research was supported by the Natural Science Foundation of Chongqing (no. cstc2019jcyj-msxmX1363) and Fundamental Research Funds for the Central Universities (no. 2018CDXYJG0040).

\section{References}

[1] D. Weideli, Environmental Analysis of US Online Shopping, MIT Center for Transportation and Logistics, Cambridge, MA, USA, 2013.

[2] W.-C. Chiang, Y. Li, J. Shang, and T. L. Urban, "Impact of drone delivery on sustainability and cost: realizing the UAV potential through vehicle routing optimization," Applied Energy, vol. 242, no. 5, pp. 1164-1175, 2019.

[3] A. A. R. Hosseinabadi, N. S. H. Rostami, M. Kardgar, S. Mirkamali, and A. Abraham, "A new efficient approach for solving the capacitated vehicle routing problem using the gravitational emulation local search algorithm," Applied Mathematical Modelling, vol. 49, no. 9, pp. 663-679, 2017.

[4] J. E. Cermak and K. Takeda, "Physical modeling of urban airpollutant transport," Journal of Wind Engineering and Industrial Aerodynamics, vol. 21, no. 1, pp. 51-67, 1985.

[5] L. Pradenas, B. Oportus, and V. Parada, "Mitigation of greenhouse gas emissions in vehicle routing problems with backhauling," Expert Systems with Applications, vol. 40, no. 8, pp. 2985-2991, 2013.

[6] J. Qian, Fuel Emission Optimization in Vehicle Routing Problems with Time-Varying Speeds, Lancaster University, Lancashire, UK, 2011. 
[7] Y.-J. Kwon, Y.-J. Choi, and D.-H. Lee, "Heterogeneous fixed fleet vehicle routing considering carbon emission," Transportation Research Part D: Transport and Environment, vol. 23, no. 8, pp. 81-89, 2013.

[8] L. Zhou, Research on the Route Optimization of Rural E-Commercial Distribution Based on "Vehicle- Unmanned Aircraft", Wuhan University of Technology, Wuhan, China, 2017.

[9] R. D’Andrea, "Guest editorial can drones deliver?" IEEE Transactions on Automation Science and Engineering, vol. 11, no. 3, pp. 647-648, 2014.

[10] Q. Y. Zhi, Research on Scheduling Mechanism of Unmanned Device in Logistics, University of Electronic Science and Technology of China, Chengdu, China, 2015.

[11] D. N. Weng, "Analysis of the main influencing factors of drone logistics distribution," Enterprise Reform and Management, vol. 23, no. 8, pp. 179-218, 2015.

[12] B. Ma, D. D. Dai, and J. R. Pan, "Regression analysis and research on influencing factors of UAVs flight stability based on MATLAB," Precise Manufacturing \& Automation, vol. 53, no. 2, pp. 32-34, 2017.

[13] C. C. Murray and A. G. Chu, "The flying sidekick traveling salesman problem: optimization of drone-assisted parcel delivery," Transportation Research Part C: Emerging Technologies, vol. 54, no. 5, pp. 86-109, 2015.

[14] Q. M. Ha, Y. Deville, Q. D. Pham, and M. H. Hà, "On the mincost traveling salesman problem with drone," Transportation Research Part C: Emerging Technologies, vol. 86, no. 1, pp. 597-621, 2018.

[15] X. Wang, S. Poikonen, and B. Golden, "The vehicle routing problem with drones: several worst-case results," Optimization Letters, vol. 11, no. 4, pp. 679-697, 2017.

[16] S. Poikonen, X. Wang, and B. Golden, "The vehicle routing problem with drones: extended models and connections," Networks, vol. 70, no. 1, pp. 34-43, 2017.

[17] L. D. P. Pugliese and F. Guerriero, "Last-mile deliveries by using drones and classical vehicles," in Proceedings of the International Conference on Optimization and Decision Science, Sorrento, Italy, September 2017.

[18] M. Pereyra and A. G. Farji-Brener, "Traffic restrictions for heavy vehicles: leaf-cutting ants avoid extra-large loads when the foraging flow is high," Behavioural Processes, vol. 170, no. 1, Article ID 104014, 2020.

[19] H. Hu, X. Li, Y. Zhang, C. Shang, and S. Zhang, "Multiobjective location-routing model for hazardous material logistics with traffic restriction constraint in inter-city roads," Computers \& Industrial Engineering, vol. 128, no. 2, pp. 861-876, 2019.

[20] B. Sureshkumar, "Review of routing algorithm for manet based on optimization techniques," Journal of the Gujarat Research Society, vol. 21, no. 17, pp. 280-285, 2019.

[21] C. Lee, "An exact algorithm for the electric-vehicle routing problem with nonlinear charging time," Journal of the Operational Research Society, vol. 71, no. 3, pp. 1-24, 2020.

[22] O. Bräysy and M. Gendreau, "Vehicle routing problem with time windows, part I: route construction and local search algorithms," Transportation Science, vol. 39, no. 1, pp. 104-118, 2005.

[23] M. Yu, V. Nagarajan, and S. Shen, "An approximation algorithm for vehicle routing with compatibility constraints," Operations Research Letters, vol. 46, no. 6, pp. 579-584, 2018.

[24] S. I. Suliman, I. Musirin, R. Dzulkifli, and R. Rifin, "The implementation of constructive heuristic algorithm for frequency assignment task in wireless communications," Advanced Science Letters, vol. 23, no. 6, pp. 5318-5323, 2017.

[25] T. Vidal, T. G. Crainic, M. Gendreau, and C. Prins, "Heuristics for multi-attribute vehicle routing problems: a survey and synthesis," European Journal of Operational Research, vol. 231, no. 1, pp. 1-21, 2013.

[26] E. Jabir, V. V. Panicker, and R. Sridharan, "Design and development of a hybrid ant colony-variable neighbourhood search algorithm for a multi-depot green vehicle routing problem," Transportation Research Part D: Transport and Environment, vol. 57, no. 12, pp. 422-457, 2017.

[27] Y. Xia, Z. Fu, S.-B. Tsai, and J. Wang, "A new TS algorithm for solving low-carbon logistics vehicle routing problem with split deliveries by backpack-from a green operation perspective," International Journal of Environmental Research and Public Health, vol. 15, no. 5, pp. 949-960, 2018.

[28] K. Peng, J. Du, F. Lu et al., "A hybrid genetic algorithm on routing and scheduling for vehicle-assisted multi-drone parcel delivery," IEEE Access, vol. 7, pp. 49191-49200, 2019.

[29] M. Moeini and H. Salewski, "A genetic algorithm for solving the truck-drone-ATV routing problem," in Proceedings of the 2019 World Congress on Global Optimization, Metz, France, July 2019.

[30] G. Laporte, "Fifty years of vehicle routing," Transportation Science, vol. 43, no. 4, pp. 408-416, 2009.

[31] O. Franzese and D. Davidson, Effect of Weight and Roadway Grade on the Fuel Economy of Class-8 Freight Trucks, Oak Ridge National Laboratory, Oak Ridge, TN, USA, 2011.

[32] A. H. Bateman, E. E. Blanco, and Y. Sheffi, "Disclosing and reporting environmental sustainability of supply chains," in Sustainable Supply Chains, pp. 119-144, Springer, Cham, Switzerland, 2017.

[33] A. Goodchild and J. Toy, "Delivery by drone: an evaluation of unmanned aerial vehicle technology in reducing $\mathrm{CO}_{2}$ emissions in the delivery service industry," Transportation Research Part D: Transport and Environment, vol. 61, no. 6, pp. 58-67, 2018.

[34] H. Duan, G. Zhang, S. Wang, and Y. Fan, "Integrated benefitcost analysis of China's optimal adaptation and targeted mitigation," Ecological Economics, vol. 160, no. 6, pp. 76-86, 2019.

[35] C. Peng, Y. Li, F. Yao et al., "Ultrahigh-energy-density fluorinated calcinated macadamia nut shell cathodes for lithium/fluorinated carbon batteries," Carbon, vol. 153, no. 11, pp. 783-791, 2019. 